\title{
The origin and non-quasiparticle nature of Fermi arcs in $\mathrm{Bi}_{2} \mathrm{Sr}_{2} \mathrm{CaCu}_{2} \mathrm{O}_{8+\delta}$
}

\author{
T. J. Reber ${ }^{1}$, N. C. Plumb ${ }^{1}$, Z. Sun ${ }^{1}$, Y. Cao', Q. Wang ${ }^{1}$, K. McElroy ${ }^{1}$, H. Iwasawa ${ }^{2}$, M. Arita ${ }^{2}$, J. S. Wen ${ }^{3}$, \\ Z. J. $\mathrm{Xu}^{3}$, G. Gu${ }^{3}$, Y. Yoshida ${ }^{4}$, H. Eisaki ${ }^{4}$, Y. Aiura ${ }^{4}$ and D. S. Dessau ${ }^{1 \star}$
}

\begin{abstract}
A Fermi $\operatorname{arc}^{1,2}$ is a disconnected segment of a Fermi surface observed in the pseudogap phase ${ }^{3,4}$ of cuprate superconductors. This simple description belies the fundamental inconsistency in the physics of Fermi arcs, specifically that such segments violate the topological integrity of the band ${ }^{5}$. Efforts to resolve this contradiction of experiment and theory have focused on connecting the ends of the Fermi arc back on itself to form a pocket, with limited and controversial success $^{6-9}$. Here we show the Fermi arc, although composed of real spectral weight, lacks the quasiparticles to be a true Fermi surface ${ }^{5}$. To reach this conclusion we developed a new photoemission-based technique that directly probes the interplay of pair-forming and pair-breaking processes with unprecedented precision. We find the spectral weight composing the Fermi arc is shifted from the gap edge to the Fermi energy by pair-breaking processes ${ }^{10}$. Although real, this weight does not form a true Fermi surface, because the quasiparticles, although significantly broadened, remain at the gap edge. This non-quasiparticle weight may account for much of the unexplained behaviour of the pseudogap phase of the cuprates.
\end{abstract}

In a solid the behaviour of the electrons is most fully described in terms of the electron Green's function, the poles of which map the energy versus momentum dependence of the electronic quasiparticles (the dressed electronic states) ${ }^{5}$. The locus of poles at the Fermi energy, $E_{\mathrm{F}}$, defines the Fermi surface of the material, from which almost all of the electronic properties of a material emanate. For a single continuous band, the Fermi surface should form a continuous loop. Consequently, the broken segments of Fermi surface known as Fermi arcs apparently require a major rethinking of some of the basic tenets of condensed matter physics.

One approach to resolve this problem is completely discarding the notion of electron quasiparticles, and with it almost all of the understanding of solids built up from generations of condensed matter physicists. Much support for this line of reasoning came from angle-resolved photoemission spectroscopy (ARPES), which is unique in its ability to directly probe the electronic excitations as a function of energy and momentum, that is the quasiparticles. Earlier ARPES studies found that the ARPES peaks were either anomalously broad or vanishingly weak ${ }^{11-13}$ especially in the underdoped 'pseudogap' regime of the cuprates_-aspects that were widely taken as evidence for the lack of electron-quasiparticles. The recent introduction of laser and low-energy ${ }^{14}$ ARPES made tremendous advances in the peak sharpness and spectral weight at $E_{\mathrm{F}}$, handicapping this line of argument. We, for example, now see sharp nodal quasiparticle-like peaks for all doping levels of $\mathrm{Bi}_{2} \mathrm{Sr}_{2} \mathrm{CaCu}_{2} \mathrm{O}_{8+\delta}$ (Bi2212) studied (down to moderately underdoped $x=0.10$ samples), in contrast to recent studies which were unable to observe peaks below $x=0.20$ (ref. 13). Therefore other ideas are needed to understand the origin of the unusual non-Fermi liquid behaviour in the cuprates. Here we show that regardless of whether the peaks are sharp enough to be considered quasiparticles, these states do not reach the Fermi energy and so cannot dominate the transport and thermodynamics in the way that regular quasiparticle states normally would. This finding reduces the relevance of the question of whether these states are sharp enough to be quasiparticles. Instead, we argue that a more relevant issue in understanding the nature of the non-Fermi liquid behaviour is whether these excitations reach the Fermi energy at all.

As well as its ability to measure quasiparticle peaks, the momentum-resolution of ARPES makes it a preferred tool to study gaps and Fermi surfaces, and therefore Fermi arcs. Despite its great power and directness, ARPES has lacked appropriate quantitative analysis techniques, which can also significantly affect the qualitative picture that emerges. For example, gap values have been predominantly measured using the approximate 'midpoint of leading edge'15 or 'peak separation of symmetrized spectra', techniques, each of which is known to fail in many limits. Phenomenological models ${ }^{16}$ have also been used to fit gapped energy distribution curves ${ }^{17}$ (EDCs), but because the EDC lineshape is not yet understood ${ }^{18}$, these fittings are not obviously better than the approximate measures.

To avoid the difficulties inherent in the traditional techniques of analysing ARPES spectra, we have developed and employed a new method, the tomographic density of states (TDoS), the creation of which is illustrated in Fig. 1 (with more details in the Supplementary Information). Briefly, the TDoS is a onedimensional momentum sum ${ }^{19}$ of the coherent electronic spectral weight (black curve in Fig. 1d), which is then normalized to a similar but ungapped reference momentum sum along the node (black curve in Fig. 1b), with some similarities to the proposal present in ref. 19. The resulting TDoS (Fig. 1f) represents the density of states and is in many ways equivalent to a typical Giaever tunnelling curve of an $s$-wave superconductor ${ }^{20}$, except that it is localized to a single slice through the band structure (hence the name tomographic, meaning sliced or sectioned). This unique advantage is indispensible when electron interactions are strongly momentum dependent (for example, in a $d$-wave superconductor).

To analyse the TDoS, we use the formula first proposed by Dynes to explain tunnelling spectra from strongly coupled $s$ wave superconductors:

$$
I_{\mathrm{TDoS}}(\omega)=\operatorname{Re} \frac{\omega-i \Gamma_{\mathrm{TDoS}}}{\sqrt{\left(\omega-i \Gamma_{\mathrm{TDoS}}\right)^{2}-\Delta^{2}}}
$$

\footnotetext{
${ }^{1}$ Department of Physics, University of Colorado, Boulder 80309-0390, USA, ${ }^{2}$ Hiroshima Synchrotron Radiation Center, Hiroshima University, Higashi-Hiroshima, Hiroshima 739-0046, Japan, ${ }^{3}$ Condensed Matter Physics and Materials Science Department, Brookhaven National Labs, Upton, New York 11973, USA, ${ }^{4}$ AIST Tsukuba Central 2, 1-1-1 Umezono, Tsukuba, Ibaraki 3058568, Japan. *e-mail: Dessau@Colorado.edu.
} 


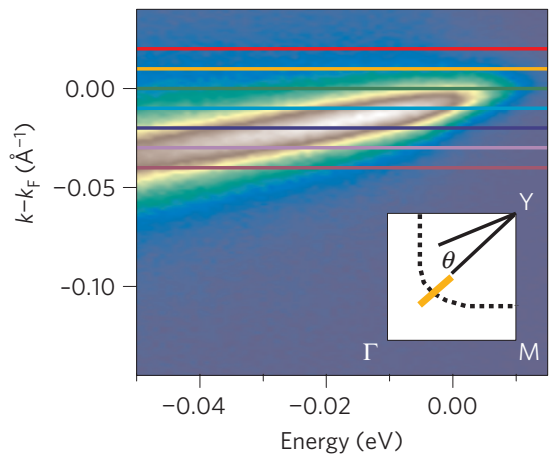

b

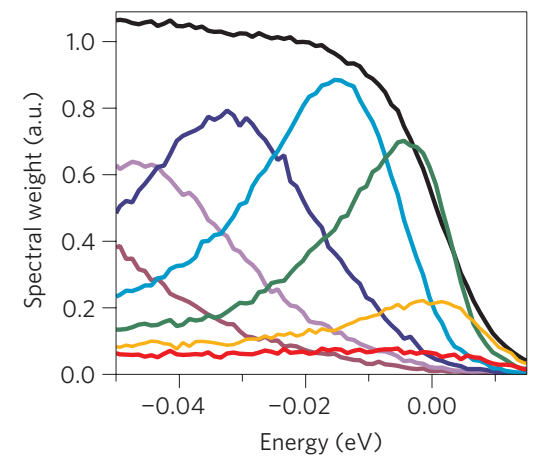

c

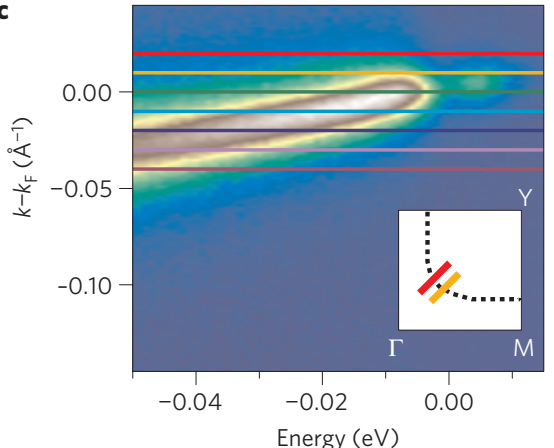

d

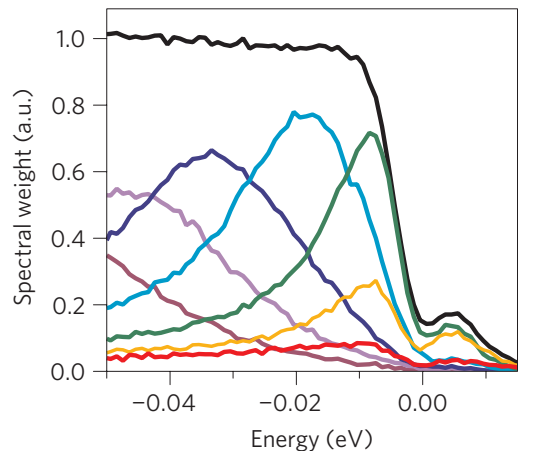

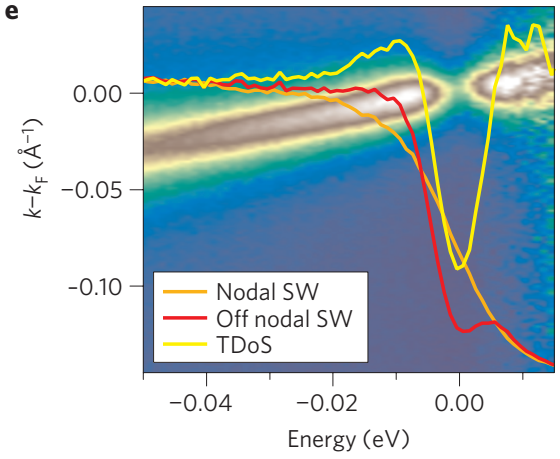

f

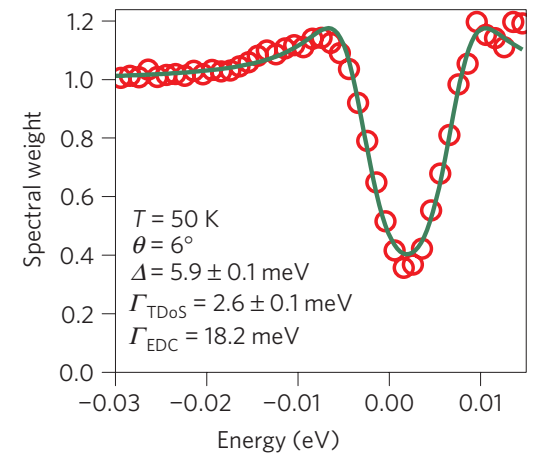

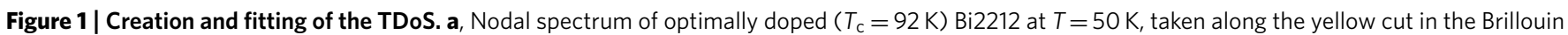
zone inset to the figure. $\mathbf{b}$, The coloured curves represent 7 out of a total of 170 individual EDCs taken along the corresponding horizontal coloured lines of a. The green EDC corresponds to $k=k_{\mathrm{F}}$. The sum of all 170 individual EDCs gives the spectral weight curve (black). c, Slightly ( $6^{\circ}$ ) off-nodal spectra taken along the red cut in the Brillouin zone inset. $\mathbf{d}$, EDCs and the corresponding spectral weight for the data of $\mathbf{c}$ showing the superconducting gap at $E_{\mathrm{F}}$ as well as the additional spectral weight above $E_{\mathrm{F}}$ due to the Bogoliubov quasiparticles. e, Off-nodal spectra normalized to nodal spectral weight (colour scale data) and the spectral weight curves from $\mathbf{b}$ and $\mathbf{d}$ (orange and red curves, respectively). The red curve is normalized to the orange curve to create the TDoS curve (yellow). f, Fit of the TDoS (red circles) to the Dynes tunnelling formula (green line).

where $I_{\mathrm{TDoS}}$ is the TDoS intensity, $\omega$ is the energy relative to $E_{\mathrm{F}}, \Gamma_{\mathrm{TDoS}}$ is the pair-breaking scattering rate and $\Delta$ is the superconducting gap $^{21}$. This formula is essentially a simple Bardeen-CooperSchrieffer density of states with gap $\Delta$, broadened by the parameter $\Gamma_{\mathrm{TDoS}}$, which is interpreted as the rate of pair-breaking. This formula has been extensively tested on conventional superconductors ${ }^{23}$ and, although initially phenomenological, it has been derived from the Eliashberg theory ${ }^{22}$. As each TDoS is specific to a single location on the Fermi surface, the gap magnitude is single valued, allowing us to use Dynes' original form even in $d$-wave superconductors. An example fit of a TDoS to equation (1) is shown in Fig. 1f, with the extracted superconducting gap value $\Delta=5.9 \pm 0.1 \mathrm{meV}$ and scattering rate $\Gamma_{\mathrm{TDos}}=2.6 \pm 0.1 \mathrm{meV}$. The $\Gamma_{\mathrm{TDos}}$ values extracted here are consistent with what has been measured by tunnelling ${ }^{24}$ and optics ${ }^{25}$, although previous ARPES-based methods to extract electronic scattering rates relied on EDC or Momentum Distribution Curve (MDC) widths that give values roughly an order of magnitude larger ${ }^{26}$ - see, for example, the EDCs of a, which have widths of order $20 \mathrm{meV}$. This difference arises because $\Gamma_{\mathrm{TDos}}$ is sensitive only to pair-breaking interactions whereas the EDCs and MDCs are sensitive to all electronic scattering processes (see Supplementary Fig. SM3).

Taking advantage of the momentum selectivity of the TDoS, Fig. 2 details the evolution of the TDoS along the Fermi surface in the near-nodal regime from an optimally doped $\left(T_{\mathrm{c}}=91 \mathrm{~K}\right) \mathrm{Bi} 2212$ sample. By fitting to the Dynes formula, equation (1), we find the gap is linear and symmetric about the node, as expected for a $d$-wave gap (Fig. 2e). In this near-nodal regime, $\Gamma_{\mathrm{TDos}}$ is essentially isotropic and of the scale of $2-3 \mathrm{meV}$, which is counter to earlier ARPES observations in which $\Gamma$ was large (of the order of $20-30 \mathrm{meV}$ ) and grew rapidly away from the node ${ }^{26}$.
In Fig. 2f, we compare the TDoS gap measurements with the EDC leading edge ${ }^{15}$ technique (data in Fig. 2c) and symmetrized EDC (refs 1,2) technique (Fig. 2d), all of which came from the identical ARPES data sets. We show $\Delta_{\text {TDos }}$ is roughly the average of the 'standard' techniques away from the node. However, the other methods both fail in proximity to the node. Specifically, both the EDC leading edge method and the symmetrized EDC methods have a short 'arc' near the node, in which the extracted gap value is either zero or negative (which is often artificially set to zero). Directly observing the sharp cusp of the $d$-wave gap so near the node confirms the findings of other probes such as thermal conductivity $^{27}$. By comparing Fig. 2e,f we see that the threshold for the artificial zero gap in the conventional techniques is when $\Gamma_{\mathrm{TDoS}} \approx \Delta$. This zero-gap regime is what previously would have been considered a Fermi arc-that is, the zero gap regime would have indicated the presence of a real portion of the Fermi surface ${ }^{2}$.

To study the Fermi arc in greater detail we move to an underdoped sample $\left(T_{\mathrm{c}}=65 \mathrm{~K}\right)$ with finely gridded momentum maps in the superconducting state $(T=10 \mathrm{~K})$ and the normal (that is, pseudogap and/or pre-pairing) state ( $T=75 \mathrm{~K}$; Fig. 3$)$. The formation of the Fermi arc is evident in the Fermi surface maps (Fig. 3a). The symmetrized EDCs show a small $\left(2^{\circ}\right)$ Fermi arc at $10 \mathrm{~K}$ but a much larger one at $75 \mathrm{~K}$, with a gap from this method (determined by the depression of weight at zero energy) only definitively present at $10^{\circ}$ and beyond (Fig. 3b). However, the TDos paint a different picture, showing a smooth evolution of the gap rather than a discrete change at any one angle (Fig. 3c). Our observation of a finite near-nodal gap above $T_{\mathrm{c}}$ is only possible if there are pre-formed superconducting pairs in the pseudogap state $e^{28,29}$.

Assuming a simple $d$-wave $\Delta$ and an isotropic $\Gamma_{\text {TDos }}$, we can fit the entire momentum dependence of the TDoS at once (Fig. $3 \mathrm{~d}$ ). 
a

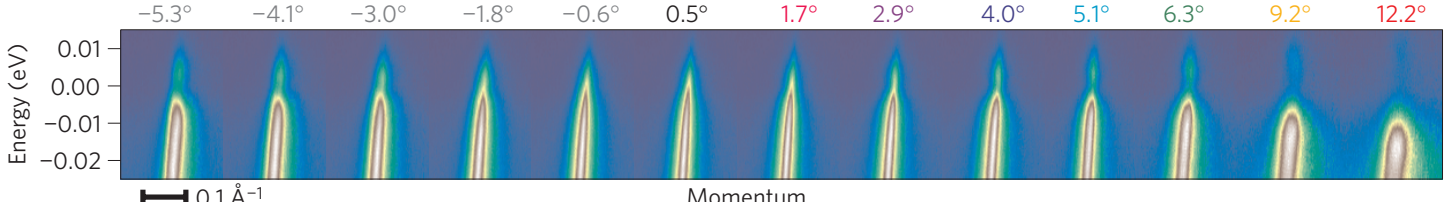

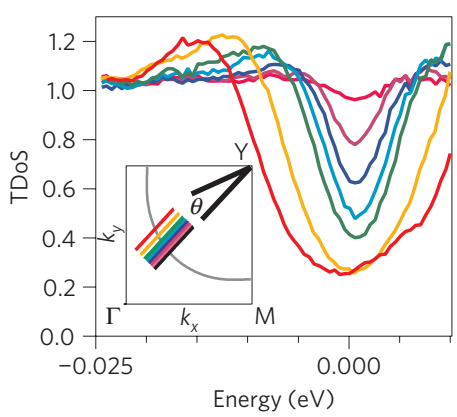

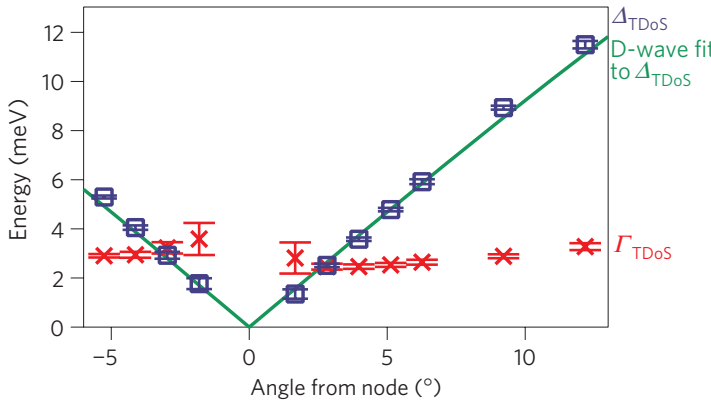

c

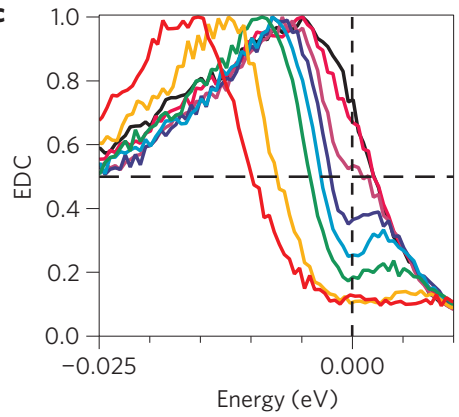

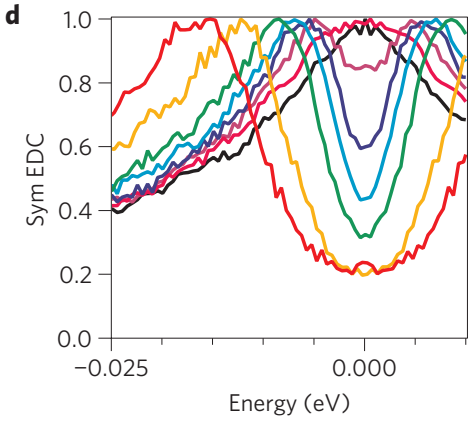

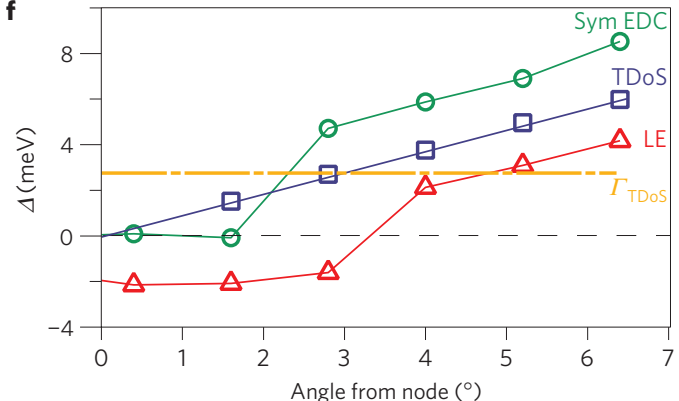

Figure 2 | Angular dependence of the TDoS and comparison with conventional ARPES analysis techniques from an optimal $T_{\mathrm{c}}=91 \mathrm{~K}$ Bi2212 sample at $\mathbf{T}=\mathbf{5 0} \mathbf{K}$. a, Angle dependence of ARPES spectra, colour coded according to location in the Brillouin zone, as indicated in the inset. $\mathbf{c}, \mathbf{d}$ follow the same colour coding, as do the positive angular labels of $\mathbf{a}$. b. TDoS curves versus angle. c, EDCs at $k_{F}$ versus angle. d, Symmetrized EDCs at $k_{F}$ versus angle, all from the same data set. e, $\Delta_{\text {TDos }}$ and $\Gamma_{\text {TDos }}$ for angles very near the node. Error bars are $\pm \sigma$ returned from fits to the data of $\mathbf{b}$. f, $\Delta_{\text {TDos }}$ near the node compared against values of $\Delta$ measured by the other two methods. These other methods show a short but artificial 'arc' of zero-gapped states near the node, whereas $\Delta_{\text {TDos }}$ extrapolates to 0 meV only at the node.
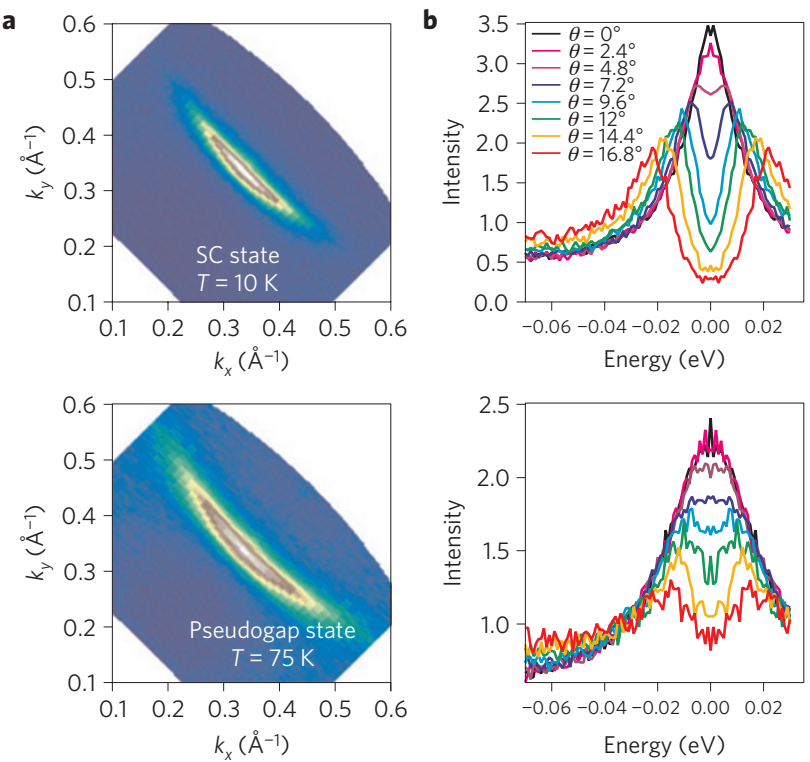
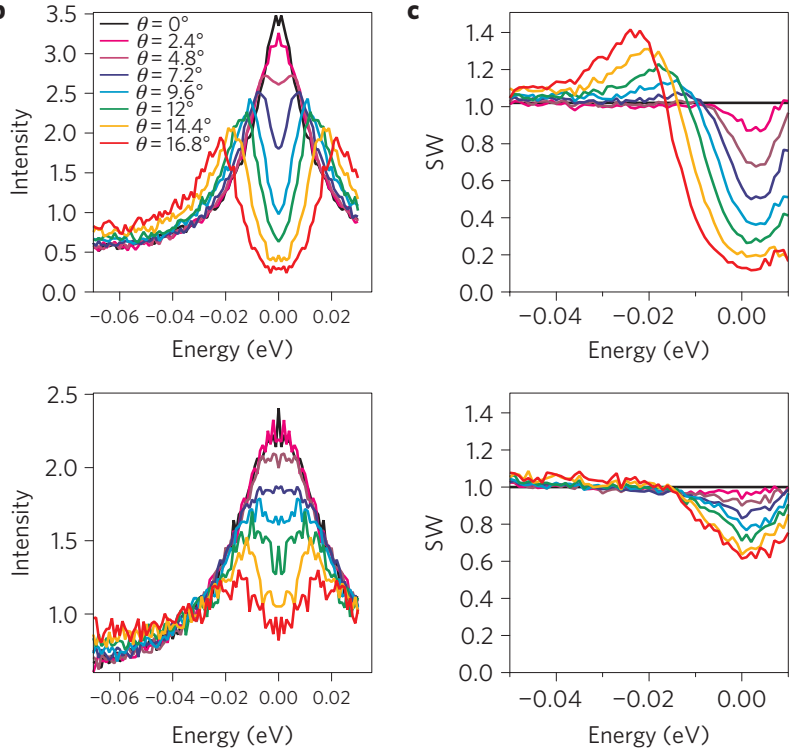
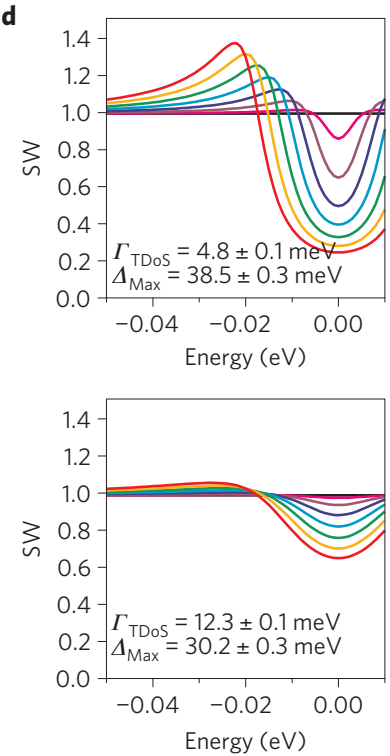

Figure 3 | Comparing the TDoS and symmetrized EDCs when determining a Fermi arc, for an underdoped $\left(T_{\mathrm{c}}=65 \mathrm{~K}\right)$ sample. a, Spectral weight at $E_{\mathrm{F}}$ both in the superconducting ( $T=10 \mathrm{~K}$, top) and pseudogap ( $T=75 \mathrm{~K}$, bottom) states. $\mathbf{b}$, Symmetrized EDCs from which one would determine a finite range of gapless states at $E_{\mathrm{F}}$ that grows with increasing temperature. The colour coding of spectra from all panels follows the angular legend shown in this panel. c, Measured TDoS spectra as a function of angle away from the node. d, Two parameter fits $\left(\Gamma_{\mathrm{TD} \text { os }}\right.$ and $\left.\Delta_{\mathrm{Max}}\right)$ for each entire set of spectra, with parameters listed on the panels. $\Delta_{\text {Max }}$ is the maximum of the $d$-wave gap, occurring at the antinode, with the $k$-dependence of the gap forced to maintain the simple $d$-wave form. $\Gamma_{\text {TDos }}$ is held to a constant value throughout $k$-space, consistent with the results of Fig. $2 \mathrm{e}$. 
a

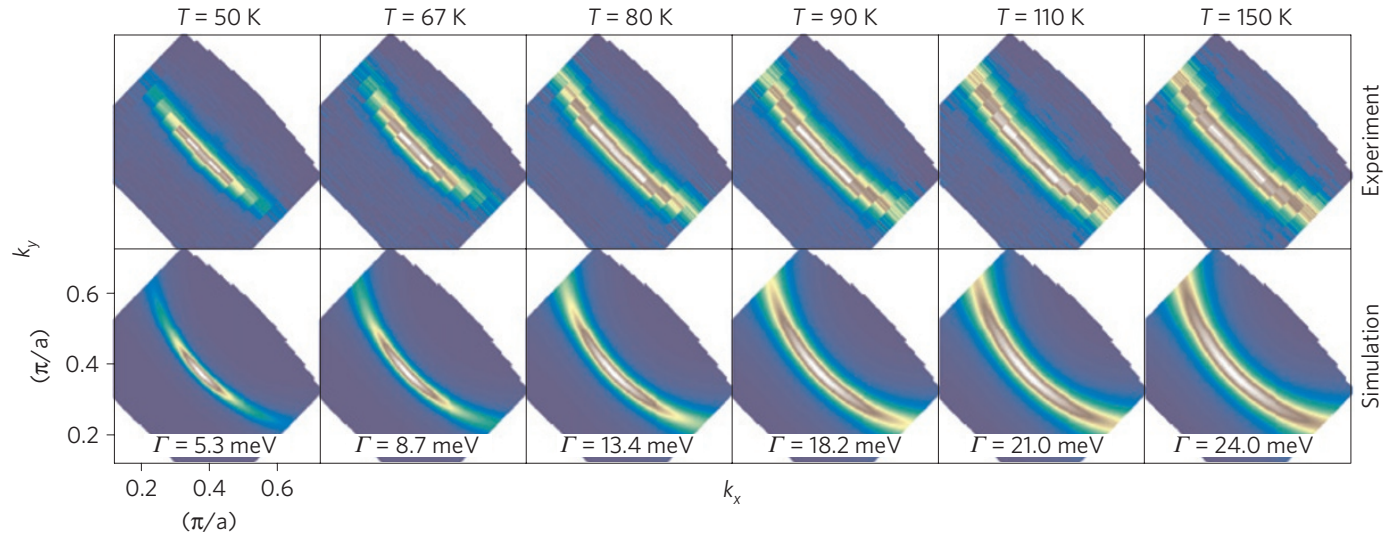

b

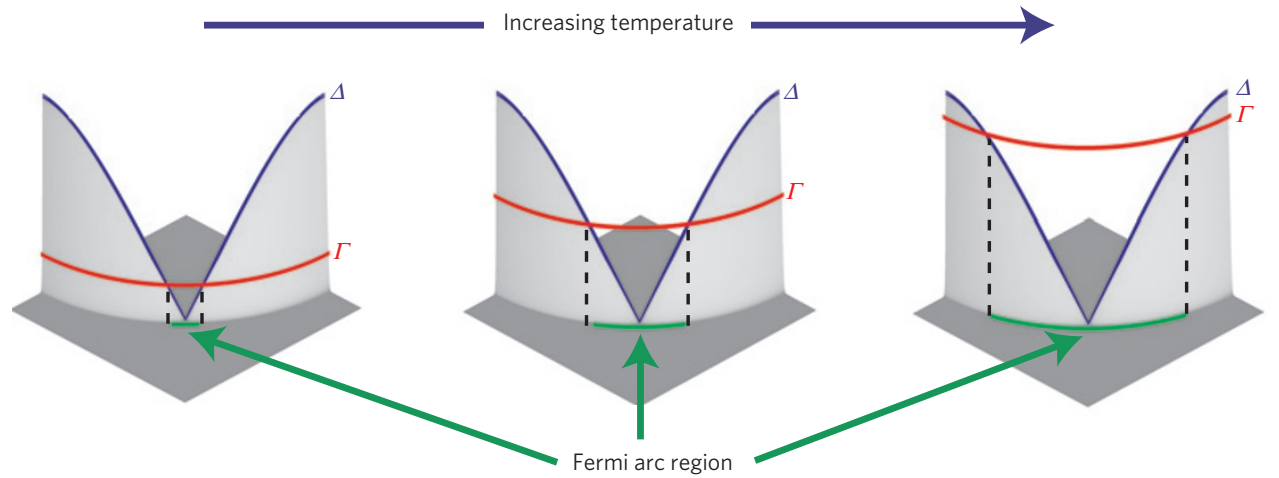

c

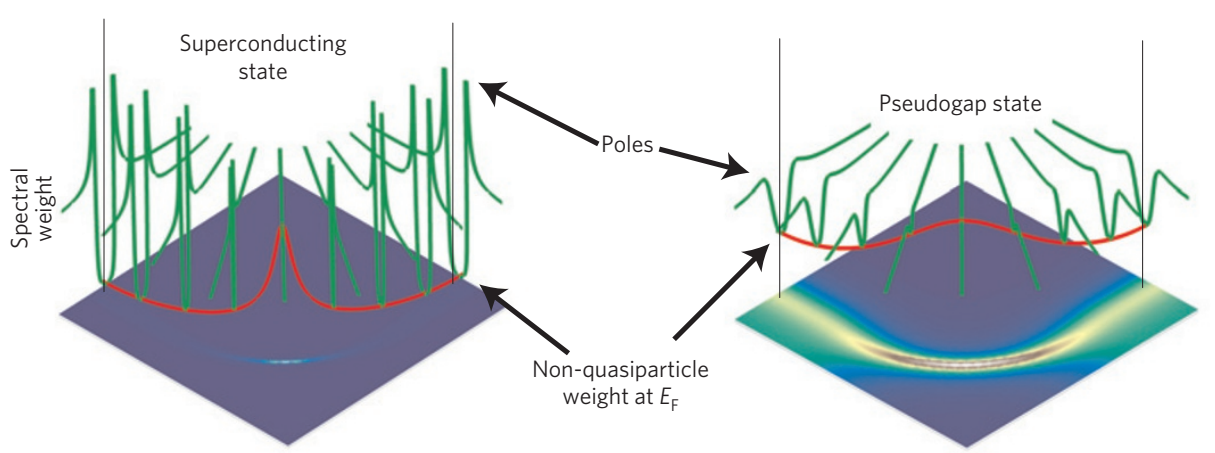

Figure 4 | Simulating the observed temperature dependence of the Fermi arc from a $T_{c}=67 \mathrm{~K}$ underdoped $\mathrm{Bi}_{2} \mathrm{Sr}_{2} \mathrm{CaCu}_{2} \mathrm{O}_{8} \mathrm{sample}_{\text {a, }}$, The unusual temperature-dependent arc length is seen in both the data and simulations. The main inputs to the simulations are an experimentally determined tight-binding band structure, a simple $d$ wave gap (both below and above $T_{\mathrm{c}}$ ) with independently measured $d$-wave gap maximum 40 meV, and a temperature-dependent scattering rate $\Gamma_{\mathrm{TD} o \mathrm{~S}}$ (shown) with units of meV. The majority of these $\Gamma_{\mathrm{TD} o \mathrm{~S}}$ values $(<110 \mathrm{~K})$ are also independently experimentally determined. $\mathbf{b}$, Simple picture for the temperature dependence of the Fermi arc length. The arc is approximately the region where $\Gamma_{\mathrm{TDoS}}>\Delta$, which grows rapidly with temperature as $\Gamma_{\mathrm{TD}}$ s grows. c, Diagram showing the change in spectral weight (red) along the Fermi surface due to increasing $\Gamma_{\mathrm{TDos}}$ between the superconducting and normal states, as determined by the TDoS (green).

Both the superconducting and pseudogap states may be fitted well with this form. Whereas $\Delta_{\text {Max }}$ has shrunk by $30 \%$ from the superconducting state to the normal state, $\Gamma_{\mathrm{TDos}}$ has more than doubled, completely filling in the gap for states close to the node. These filled-in states are the source of previous observations of a Fermi arc and should be considered a manifestation of superconductive pre-pairing in the pseudogap state. The extremely short arc in the superconducting state is most probably due to in-plane impurity scattering (for example, $\mathrm{Cu}$ vacancies) ${ }^{30,31}$. However, the growth of the arc with temperature cannot be explained by static disorder. Instead, a dynamical process must lead to the increased pair-breaking rate that causes the arc to grow ${ }^{10}$, as was first proposed in ref. 10.

A more complete temperature dependence of the Fermi arc is shown in Fig. 4a, for a similarly underdoped sample $\left(T_{\mathrm{c}}=67 \mathrm{~K}\right)$.
The growth of the Fermi arc with temperature ${ }^{2}$ can be accurately simulated with an electron Green's function which uses a tight-binding band structure obtained from experiment, a simple $d$-wave gap $\left(\Delta_{\mathrm{Max}}=38 \mathrm{meV}\right)$, and a scattering rate $\Gamma_{\mathrm{TD} \text { os }}$ which is constant across the Fermi surface (Fig. 2e) but which varies strongly with temperature. The values of the electron scattering rate are directly obtained from the Dynes fits up to $110 \mathrm{~K}$. At higher temperatures the fits become less reliable, so we have extrapolated those values. Although the gap is seen to close slightly with increasing temperature (Fig. 3d), it was held constant in Fig. 4 for simplicity. The observed growth of the Fermi arc with increased temperature can be understood with the aid of the schematic of Fig. 4b. As the dominant effect of raising temperature is to increase $\Gamma_{\mathrm{TDos}}$, the threshold of $\Gamma_{\mathrm{TDos}}=\Delta$ moves away from the node, increasing the arc length. 
The notion of discontinuous Fermi arcs put forward from the previous ARPES experiments is unphysical within the context of standard condensed matter theory, because Fermi surfaces must be topologically connected. Therefore, a great effort has been made to observe if and how these arcs close to form a Fermi pocket. For example, recent ARPES experiments ${ }^{6,7}$ have presented evidence for arc closures, although many of these have been met with scepticism for reasons such as including potential contamination from shadow bands, superstructure bands ${ }^{9}$, or extended extrapolations. With the understanding presented here, there is no physical reason why the arc would need to be closed, because it is not a real locus of quasiparticle states. In this light, the difficulty in observing the 'back' side of the arc is completely natural — that is, the arc is actually arclike, as opposed to only being the front side of a small hole pocket.

High magnetic field quantum oscillation studies in the cuprates have found evidence for a small Fermi surface pocket ${ }^{8}$, with these pockets potentially related to the pockets discussed in some of the ARPES experiments. However, Hall effect measurements indicate that the pockets hold electrons instead of holes ${ }^{8}$, making it clear that the pockets observed in the quantum oscillation experiments are inconsistent with the ARPES Fermi arc with backside closure. Furthermore, new evidence from nuclear magnetic resonance experiments indicates that the high magnetic fields used in the quantum oscillation experiments cause a reconstruction of the Fermi surface that can create the small pockets observed in those experiments ${ }^{32}$.

Within the conventional analysis method of symmetrized EDCs, the Fermi arcs are considered loci of quasiparticle states at the Fermi level that grow with increasing temperature. Instead, the above simulations and data argue that the arcs are a result of the interplay between $\Delta(k, T)$ and $\Gamma_{\mathrm{TDoS}}(k, T)$, which we find is dominated by $\Delta(k)$ and $\Gamma_{\mathrm{TDos}}(T)$. Importantly, this result indicates that the arcs are not composed of true quasiparticles, because, even though the quasiparticles exist, they are not at at $E_{\mathrm{F}}-$ rather the pole locations are the gap values, $\Delta(k)$ (Fig. $4 \mathrm{c}$ ). Rather than a true Fermi surface of quasiparticle poles, the arcs are regions where real spectral weight has been scattered inside the $d$-wave gap, with this incoherent non-quasiparticle weight varying with Fermi surface angle and temperature (Fig. 4c). Aside from the single quasiparticle state at the node, this non-quasiparticle weight comprises the only states available to contribute to the low-energy transport and thermodynamic properties. Therefore, these states are strong candidates to explain the unusual transport, heat capacity, and other thermodynamic properties in the pseudogap state of the cuprates ${ }^{33}$.

Received 16 February 2012; accepted 24 May 2012; published online 1 July 2012

\section{References}

1. Norman, M. et al. Destruction of the Fermi surface in underdoped high- $T_{\mathrm{c}}$ superconductors. Nature 392, 157-160 (1998).

2. Kanigel, A. et al. Evolution of the pseudogap from Fermi arcs to the nodal liquid. Nature Phys. 2, 447-451 (2006).

3. Loeser, A. G. et al. Excitation gap in the normal state of underdoped $\mathrm{Bi}_{2} \mathrm{Sr}_{2} \mathrm{CaCu}_{2} \mathrm{O}_{8+\delta}$. Science 273, 325-329 (1996).

4. Ding, H. et al. Spectroscopic evidence for a pseudogap in the normal state of underdoped high- $T_{\mathrm{c}}$ superconductors. Nature 382, 51-54 (1996).

5. Pines, D. \& Nozieres, P. The Theory of Quantum Liquids Vol. 1 (W.A. Benjamin, 1966)

6. Yang, H-B. et al. Emergence of preformed Cooper pairs from the doped Mott insulating state in $\mathrm{Bi}_{2} \mathrm{Sr}_{2} \mathrm{CaCu}_{2} \mathrm{O}_{8+\delta}$. Nature 456, 77-80 (2008).

7. Meng, J. et al. Coexistence of Fermi arcs and Fermi pockets in a high- $T_{\mathrm{c}}$ copper oxide superconductor. Nature 462, 335-338 (2009).

8. Doiron-Leyraud, N. et al. Quantum oscillations and the Fermi surface in an underdoped high- $T_{\mathrm{c}}$ superconductor. Nature 447, 565-568 (2007).

9. King, P. D. C. et al. Structural origin of apparent Fermi surface pockets in angle-resolved photoemission of $\mathrm{Bi}_{2} \mathrm{Sr}_{2-x} \mathrm{La}_{x} \mathrm{CuO}_{6+\delta}$. Phys. Rev. Lett. 106, 127005 (2011).

10. Norman, M. R., Kanigel, A., Randeria, M., Chatterjee, U. \& Campuzano, J. C. Modeling the Fermi arc in underdoped cuprates. Phys. Rev. B 76, 174501 (2007)
11. Damascelli, A., Hussain, Z. \& Shen, Z-X. Angle-resolved photoemission studies of the cuprate superconductors. Rev. Mod. Phys. 75, 473-541 (2003).

12. Laughlin, R. B. Evidence for quasipartile decay from photoemission in underdoped cuprates. Phys. Rev. Lett. 79, 1726-1729 (1997).

13. Fournier, D. et al. Loss of nodal quasiparticle integrity in underdoped $\mathrm{YBa}_{2} \mathrm{Cu}_{3} \mathrm{O}_{6+x}$. Nature Phys. 6, 905-911 (2010).

14. Koralek, J. D. et al. Laser based angle-resolved photoemission, the sudden approximation, and quasiparticle-like spectral peaks in $\mathrm{Bi}_{2} \mathrm{Sr}_{2} \mathrm{CaCu}_{2} \mathrm{O}_{8+\delta}$. Phys. Rev. Lett. 96, 017005 (2006).

15. Shen, Z-X. et al. Anomalously large gap anisotropy in the a-b plane of $\mathrm{Bi}_{2} \mathrm{Sr}_{2} \mathrm{CaCu}_{2} \mathrm{O}_{8+}$. Phys. Rev. Lett. 70, 1553-1556 (1993).

16. Norman, M. R., Randeria, M., Ding, H. \& Campuzano, J. C. Phenomenology of the low-energy spectral function in high- $T_{\mathrm{C}}$ superconductors. Phys. Rev. $B$ 57, R11093-R11096 (1998).

17. Lee, W. S. et al. Abrupt onset of a second energy gap at the superconducting transition of underdoped Bi2212. Nature 450, 81-84 (2007).

18. Casey, P. A., Koralek, J. D., Plumb, N. C., Dessau, D. S. \& Anderson, P. W. Accurate theoretical fits to laser-excited photoemission spectra in the normal phase of high temperature superconductors. Nature Phys. 4, 210-212 (2008).

19. Vehkter, I. \& Varma, C. M. Proposal to determine the spectrum of pairing glue in high-temperature superconductors. Phys. Rev. Lett. 90, 237003 (2003).

20. Giaever, I., Hart, H. R. \& Mergele, K. Tunneling into superconductors at temperatures below 1 K. Phys. Rev. 126, 941-948 (1962).

21. Dynes, R. C., Narayanamurti, V. \& Garno, J. P. Direct measurement of quasiparticle-lifetime broadening in a strong-coupled superconductor. Phys. Rev. Lett. 41, 1509-1512 (1978).

22. Mikhailovsky, A. A., Shulga, S. V., Karakozov, A. E., Dolgov, O. V. \& Maksimov, E. G. Thermal pair-breaking in superconductors with strong electron-phonon interaction. Solid State Commun. 80, 511-515 (1991).

23. Wolf, E. L. Principles of Electron Tunneling Spectroscopy (Oxford Univ. Press, 1989).

24. Pasupathy, A. et al. Electronic origin of the inhomogeneous pairing interaction in the high- $T_{\mathrm{c}}$ superconductor $\mathrm{Bi}_{2} \mathrm{Sr}_{2} \mathrm{CaCu}_{2} \mathrm{O}_{8+\delta}$. Science 320, 196-201 (2008).

25. Hwang, J., Timusk, T. \& Gu, G. D. High-transition-temperature superconductivity in the absence of the magnetic-resonance mode. Nature 427, 714-717 (2004).

26. Valla, T. et al. Temperature dependent scattering rates at the Fermi surface of optimally doped $\mathrm{Bi}_{2} \mathrm{Sr}_{2} \mathrm{CaCu}_{2} \mathrm{O}_{8+\delta}$. Phys. Rev. Lett. 85, 828-831 (2000).

27. Sutherland, M. et al. Thermal conductivity across the phase diagram of cuprates: Low-energy quasiparticles and doping dependence of the superconducting gap. Phys. Rev. B 67, 174520 (2003).

28. Emery, V. J. \& Kivelson, S. A. Importance of phase fluctuations in superconductors with small superfluid density. Nature 374, 434-437 (1995).

29. Wang, Y., Li, L. \& Ong, N. P. Nernst effect in high $T_{c}$ superconductors. Phys. Rev. B 73, 024510 (2006).

30. Fehrenbacher, R. Effect of nonmagnetic impurities on the gap of a d $\mathrm{d}_{x^{2}-y^{2}}$ superconductor as seen by angle-resolved photo-emission. Phys. Rev. B 54, 6632-6639 (1996)

31. Haas, S., Balatsky, A. V., Sigrist, M. \& Rice, T. M. Extended gapless regions in disordered $\mathrm{d}_{x^{2}-y^{2}}$ wave superconductors. Phys. Rev. B 56, 5108-5111 (1997).

32. $\mathrm{Wu}, \mathrm{T}$. et al. Magnetic-field-induced charge-stripe order in the high-temperature superconductor $\mathrm{YBa}_{2} \mathrm{Cu}_{3} \mathrm{Oy}$. Nature 477, 191-194 (2011).

33. Varma, C. M., Littlewood, P. B., Schmitt-Rink, S., Abrahams, E. \& Ruckenstein, A. E. Phenomenology of the normal state of $\mathrm{Cu}-\mathrm{O}$ high-temperature superconductors. Phys. Rev. Lett. 63, 217-218 (1989).

\section{Acknowledgements}

We thank G. Arnold, A. Balatsky, I. Mazin, T. Senthil and M. Hermele for valuable conversations and D. H. Lu and R. G. Moore for help at the Stanford Synchrotron Radiation Laboratory (SSRL). SSRL is operated by the Department of Energy, Office of Basic Energy Sciences. ARPES experiments at the Hiroshima Synchrotron Radiation Center were performed under proposal 09-A-48. Funding for this research was provided by DOE Grant No. DE-FG02-03ER46066 (Colorado) and DE-AC02-98CH10886 (Brookhaven) with partial support from the National Science Foundation EUV Engineering Research Center and from Kakenhi (10015981 and 19340105).

\section{Author contributions}

T.J.R., N.C.P., Z.S., Y.C., Q.W., M.A. and H.I. performed the ARPES measurements. J.S.W., Z.J.X., G.G., Y.Y., H.E. and Y.A. grew and prepared the samples. T.R. analysed the ARPES data. T.J.R and D.S.D. developed the TDoS technique and wrote the paper with suggestions and comments by N.C.P., Z.S., K.M. and all other authors. ARPES simulations were done by T.J.R. D.S.D. is responsible for project direction, planning and infrastructure.

\section{Additional information}

The authors declare no competing financial interests. Supplementary information accompanies this paper on www.nature.com/naturephysics. Reprints and permissions information is available online at www.nature.com/reprints. Correspondence and requests for materials should be addressed to D.S.D. 